\title{
(3R)-5,6,7-trihydroxy-3-isopropyl-3-methylisochroman-1-one attenuates cardiac dysfunction via the apelin/APJ signaling pathway
}

\author{
MEI DING ${ }^{1}$, LIANYUE GUAN ${ }^{2}$, CHUNMEI ZHANG ${ }^{3}$, PING YANG ${ }^{1}$ and HAILING YANG ${ }^{3}$ \\ Departments of ${ }^{1}$ Cardiology, ${ }^{2}$ Hepatobiliary-Pancreatic Surgery and ${ }^{3}$ Emergency, \\ China-Japan Union Hospital of Jilin University, Changchun, Jilin 130033, P.R. China
}

Received June 14, 2018; Accepted March 26, 2019

DOI: $10.3892 / \mathrm{mmr} .2019 .10122$

\begin{abstract}
Myocardial infarction (MI) is associated with a high risk of mortality and is a major global health concern. The present study aimed to investigate the protective effects of (3R)-5,6,7-trihydroxy-3-isopropyl-3-methylisochroman-1-one (TIM) against MI induced by isoproterenol (ISO) in a rat model and the underlying mechanisms. Wistar rats were assigned to 4 groups $(n=10)$ : The control group received saline treatment; the ISO group received an intraperitoneal injection of ISO (100 mg/kg); and the TIM (low) and TIM (high) groups received an intraperitoneal injection of ISO, plus a 1 and $2 \mathrm{mg} / \mathrm{kg}$ dose of TIM orally, respectively. TIM rats were treated with TIM daily for 12 days and received ISO injections on the final 2 days to induce MI. Cardiac function, apoptosis index and protein expression were subsequently determined. The levels of oxidative stress markers were determined by ELISAs, whereas DNA damage was detected using a Cell Death Detection ELISA kit. Gene and protein expression were determined via reverse transcription-quantitative polymerase chain reaction and western blot analyses, respectively. Following treatment with ISO, the maximum left ventricular contraction/relaxation velocity and left ventricular systolic pressure were significantly decreased, whereas the left ventricular end-diastolic pressure was increased; however, treatment with TIM significantly ameliorated ISO-induced cardiac dysfunction. Additionally, TIM treatment significantly
\end{abstract}

Correspondence to: Dr Hailing Yang, Department of Emergency, China-Japan Union Hospital of Jilin University, 126 Xiantai Street, Changchun, Jilin 130033, P.R. China

E-mail: yanghailing2016@163.com

Dr Ping Yang, Department of Cardiology, China-Japan Union Hospital of Jilin University, 126 Xiantai Street, Changchun, Jilin 130033, P.R. China

E-mail: pyang@jlu.edu.cn

Key words: myocardial infarction, (3R)-5,6,7-trihydroxy-3isopropyl-3-methylisochroman-1-one, cardiomyocyte apoptosis, apelin, apelin receptor decreased oxidative stress and inhibited the apoptosis of cardiomyocytes, as determined by a decrease in caspase activities, increased expression of B-cell lymphoma 2 (Bcl-2) and reduced expression of cleaved caspase-3, cleaved caspase- 9 and Bcl-2-associated X. Furthermore, treatment with TIM upregulated the levels of apelin in the plasma and myocardium of ISO-treated rats. The results indicated that TIM protected cardiomyocytes against ISO-induced MI, potentially via the apelin/apelin receptor signaling pathway. The results of the present study suggested that TIM may be a potential novel therapy for the treatment of MI.

\section{Introduction}

Myocardial infarction (MI) is one of the leading causes of cardiac-associated mortality globally, and is accompanied by cardiomyocyte apoptosis, inflammation and cardiac fibrosis, leading to an increased risk of adverse cardiac events and eventual heart failure (1-3). Clinical management of MI has notably improved; however, MI and the associated complications remain major causes of morbidity and mortality (4). Therefore, the identification of novel therapeutic strategies is important for improving cardiac function following MI.

Isoproterenol (ISO), a synthetic catecholamine and $\beta$-adrenergic agonist, is frequently used in preclinical studies to induce MI in rats (5). Treatment with ISO induces severe oxidative stress in the myocardium and subsequent infarct-like necrosis of the heart muscles in rats, which is accompanied by decreased cardiac function and the increased apoptosis of cardiomyocytes $(6,7)$. The ISO-induced rat model of MI has been widely validated and exhibits the greatest similarity to the symptoms of MI in clinical settings (8). This model of MI has been extensively used to investigate potential cardioprotective drugs $(9,10)$.

Identified in 1998, apelin is the endogenous ligand of the G-protein-coupled apelin receptor (APJ) and is expressed in various organs, including the heart, lung, liver and brain (11). In clinical settings, the plasma levels of apelin have been reported to decrease in patients with cardiac dysfunction $(12,13)$; however, patients with a ventricular assist device exhibited marked increases in apelin levels in the left ventricle (14). A previous study demonstrated that the apelin/APJ signaling 
pathway was involved in the maintenance of cardiac function; apelin treatment protected the heart in a rat model of ischemia/reperfusion injury (15). Therefore, the apelin/APJ signaling pathway may be a novel target in the treatment of heart failure.

There has been a notable increase in the use of herbs and their extracts to treat diseases in previous decades $(16,17)$. A novel compound isolated from Alpinia katsumadai Hayata, (3R)-5,6,7-trihydroxy-3-isopropyl-3-methylisochroman-1-one (TIM; Fig. 1A) exhibited potent cardioprotective effects in a recent study, reducing lipoteichoic acid-induced damage in rat cardiomyoblast cells via the inhibition of oxidative stress (18). Further investigation into the cardioprotective efficacy of TIM may reveal the compound to be a potential therapy in the treatment of cardiovascular diseases. The aim of the present study was to determine the effects of TIM on ISO-induced cardiac dysfunction in rats and the underlying mechanisms.

\section{Materials and methods}

Materials. A total of 50 male Wistar rats (3-4 months old, 180-220 g) were purchased from Beijing Vital River Laboratory Animal Technology, Co., Ltd. (Beijing, China), provided with ad libitum access to food and water, and housed at $21 \pm 2{ }^{\circ} \mathrm{C}$ with $60 \pm 5 \%$ humidity under a standard 12-h light/dark cycle. All animal experiments were performed in accordance with the Chinese Legislation on the Use and Care of Laboratory Animals (19), and approved by the Ethical Committee on Animal Care and Use of Jilin University (Changchun, China). Lactate dehydrogenase (LDH; cat. no. A020-2), malondialdehyde (MDA; cat. no. A003-1), glutathione (GSH; cat. no. A006-2) and superoxide dismutase (SOD; cat. no. A001-3) assay kits were purchased from Jiancheng Bioengineering Institute (Nanjing, China). Caspase-3/9 activity assay kits [cat. no. CASP3C (Caspase-3); cat. no. APT173 (Caspase-9)] and ISO (cat. no. 1351005) were purchased from Sigma-Aldrich (Merck KGaA, Darmstadt, Germany). A Cell Death Detection ELISAplus kit (cat. no. 11544675001) was purchased from Roche Applied Science (Penzberg, Germany) to determine DNA fragmentation. A Cytochrome-c assay kit (cat. no. MCTC0) was purchased from R\&D Systems, Inc. (Minneapolis, MN, USA). An apelin-12 immunoassay kit (cat. no. EK-057-23) was obtained from Phoenix Pharmaceuticals Inc. (Belmont, CA, USA). Reverse transcription-quantitative polymerase chain reaction (RT-qPCR) Power SYBR ${ }^{\circledR}$ Green Master Mix (cat. no. 4367659) was purchased from Thermo Fisher Scientific, Inc. (Waltham, MA, USA). TIM was isolated and identified by Professor Lin from Shantou University Medical College (Shantou, China), and kindly provided by Professor Lin for use in the present study (20). Sodium carboxymethyl cellulose (CMC-Na) is widely used in the food and pharmaceutical industries due to its high viscosity and minimal toxicity (21); TIM was suspended in CMC-Na (Changshu Wealthy Science and Technology Co., Ltd, Changshu, China) prior to treatment. The doses of TIM used in the present study were determined based on a preliminary study. In the preliminary study, the protective activities of TIM were investigated using five doses $(0.5,1,2,5$ and $10 \mathrm{mg} / \mathrm{kg} ; \mathrm{n}=3 / \mathrm{group})$; it was revealed that $0.5 \mathrm{mg} / \mathrm{kg}$ TIM possessed no protective effects, whereas 5 and $10 \mathrm{mg} / \mathrm{kg}$ TIM induced weight loss in addition to improving cardiac function in the ISO-induced MI model (data not shown). TIM (1 or $2 \mathrm{mg} / \mathrm{kg}$ ) effectively protected against ISO-induced heart dysfunction without effects on body or heart weight. Therefore, 1 and $2 \mathrm{mg} / \mathrm{kg}$ were selected for subsequent experiments. All other chemicals used in the present study were of analytical grade and purchased from Sinopharm Chemical Reagent Co., Ltd. (Shanghai, China).

Experimental procedures. Acute MI was induced via daily intraperitoneal injection of ISO $(100 \mathrm{mg} / \mathrm{kg})$ into rats for 2 consecutive days. Rats were randomly assigned to four groups ( $n=10 /$ group) and treated for 12 days: The normal control group was treated with saline orally for 12 days and by intraperitoneal injection for the final 2 days; the ISO group was treated with saline orally for 12 days and injected with ISO on the final 2 days; and the TIM (low) and TIM (high) groups, were treated daily with TIM (1 and $2 \mathrm{mg} / \mathrm{kg}$, respectively) orally for 12 days and then injected with ISO on the final 2 days. Body weight was measured every 2 days.

Measurement of heart function. Blood pressure was recorded $48 \mathrm{~h}$ following the first ISO injection using a computerized, non-invasive tail-cuff system. Rats were subsequently anesthetized using a mixture of ketamine $(40 \mathrm{mg} / \mathrm{kg})$, xylazine $(8 \mathrm{mg} / \mathrm{kg})$ and acepromazine $(1 \mathrm{mg} / \mathrm{kg})$, and left ventricular function was measured by inserting a heparin-filled catheter $(500 \mathrm{U} / \mathrm{ml})$ into the left ventricle. Left ventricular systolic pressure (LVSP), left ventricular end-diastolic pressure (LVEDP) and maximum left ventricular contraction/relaxation velocity $\left( \pm \mathrm{LV} \mathrm{dp} / \mathrm{dt}_{\max }\right)$ were recorded using a BL-420E monitor system.

Sample collection. Following measurement of cardiac function, blood samples $(500 \mu \mathrm{l})$ were collected from the hearts of anesthetized rats. Rats were subsequently sacrificed via inhalation of $\mathrm{CO}_{2}$ for a minimum of 5 min using a flow rate of $21 / \mathrm{min}$ in a $10 \mathrm{l}$ chamber. Rats were kept in the chamber until a heartbeat could no longer be felt. Mortality was confirmed by removal of the heart. Following sacrifice, the heart weight was recorded, and myocardial tissues from the injured areas of the hearts were collected and washed with ice-cold physiological saline for further analysis. The heart index was defined as the heart weight/body weight ratio.

Immunoassay measurement. The myocardial tissues were homogenized on ice. The homogenate was centrifuged at $6,000 \mathrm{x} \mathrm{g}$ for $20 \mathrm{~min}$ at $4^{\circ} \mathrm{C}$, the supernatant was collected and the protein concentration was quantified using the Bradford assay. Apelin, cytochrome-c, LDH, MDA, SOD and GSH were measured with the corresponding assay kits according to the manufacturers' protocols.

DNA fragmentation assay. DNA fragmentation was determined using a Cell Death Detection ELISAplus kit. The myocardial tissues were lysed for $30 \mathrm{~min}$ at room temperature, and then the homogenate was centrifuged for $10 \mathrm{~min}$ at $2,000 \times \mathrm{g}$ at $4^{\circ} \mathrm{C}$. Supernatant $(20 \mu \mathrm{l})$ was incubated with a mixture of anti-DNA-peroxidase and anti-histone-biotin for $30 \mathrm{~min}$ at room temperature. Following the addition of 
A<smiles>[3H]C1(C(C)C)Cc2c(cc(O)c(O)c2O)C(=O)O1</smiles>

C

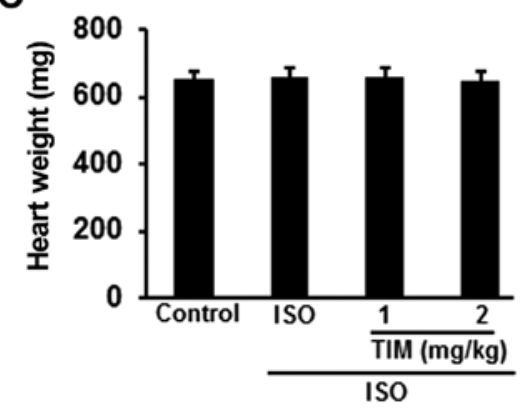

B

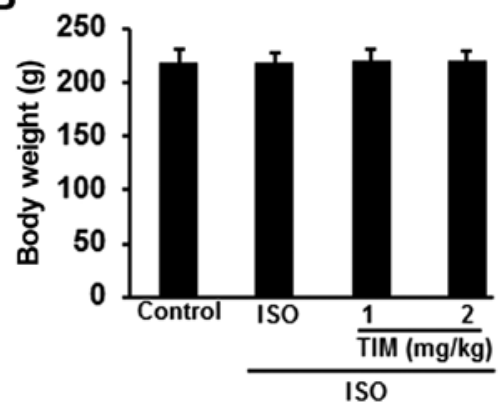

D

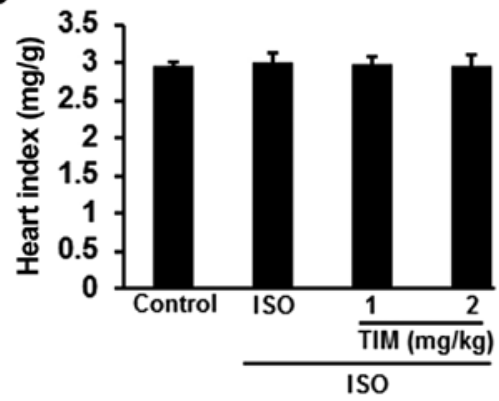

Figure 1. Effects of MI and TIM on body and heart weight. (A) Chemical structure of TIM. (B) Body weight following injection with saline or ISO and oral treatment of saline or TIM (1 or $2 \mathrm{mg} / \mathrm{kg}$ ). (C) Heart weight following ISO-induced MI and treatment with TIM. (D) Heart index (heart weight/body weight) following ISO-induced MI and treatment with TIM. No significant differences in body weight, heart weight or heart index were observed across groups. Data are presented as the mean \pm standard deviation. Samples were measured in triplicate. Data distributions were analyzed using a Kolmogorov-Smirnov test. ISO, isoproterenol; MI, myocardial infarction; TIM, (3R)-5,6,7-trihydroxy-3-isopropyl-3-methylisochroman-1-one.

2,2'-azino-bis (3-ethylbenzthiazoline-6-sulfonic acid) as the substrate for $20 \mathrm{~min}$ at room temperature, the levels of peroxidase in the immunocomplex were quantified. The absorbance at $405 \mathrm{~nm}$ was detected using a microplate reader.

Caspase activity measurement. The homogenate from myocardial tissues was analyzed for caspase- 3 and caspase- 9 activity using assay kits, according to the manufacturer's protocols.

$R T$ - $q P C R$. Total RNA was extracted from myocardial tissues using TRIzol ${ }^{\circledR}$ (Thermo Fisher Scientific, Inc.). mRNA was then reverse transcribed into cDNA using the iScript ${ }^{\mathrm{TM}}$ Reverse Transcription Supermix kit (Bio-Rad Laboratories, Inc., Hercules, CA, USA). RT was conducted as follows: $25^{\circ} \mathrm{C}$ for $5 \mathrm{~min} ; 46^{\circ} \mathrm{C}$ for $20 \mathrm{~min}$; and $95^{\circ} \mathrm{C}$ for $1 \mathrm{~min}$ ). $\mathrm{qPCR}$ was performed using $\mathrm{SYBR}^{\circledR}$ Green Supermix (Bio-Rad Laboratories, Inc) as follows: $95^{\circ} \mathrm{C}$ for $10 \mathrm{~min}$, and 40 cycles of $95^{\circ} \mathrm{C}$ for $15 \mathrm{sec}$ and $60^{\circ} \mathrm{C}$ for $60 \mathrm{sec}$. The expression levels of target genes were determined using the $2^{-\Delta \Delta \mathrm{Cq}}$ method (22). mRNA expression was normalized to the housekeeping gene $\beta$-actin. The gene-specific primer sequences were as follows: Apelin, forward, 5'-GTGAAGCCCAGAACTTCGAG-3' and reverse, 3'-CAGCGATAACAGGTGCAAGA-5'; APJ, forward, 5'-TGTACGCCAGTGTCTTTTGC-3' and reverse, 3'-CTG TTTTCCGGGATGTCAGT-5'; and $\beta$-actin, forward, 5'-AGC CATGTACGTAGCCATCC-3' and reverse, 3'-CTCTCAGCT GTGGTGGTGAA-5'. The experiment was repeated three times.

Western blotting. Total cellular and nuclear protein was extracted from the myocardial tissues using NE-PER ${ }^{\mathrm{TM}}$ Nuclear and Cytoplasmic Extraction Reagents (Thermo Fisher Scientific,
Inc.). Protein concentration was determined using the Bradford method. Following boiling, protein (50 $\mu \mathrm{g} /$ lane) was separated via $4-12 \%$ SDS-PAGE and then transferred onto polyvinylidene difluoride membranes. The membranes were blocked with $1 \%$ bovine serum albumin (cat. no. A9306; Sigma-Aldrich; Merck $\mathrm{KGaA}$ ) for $1 \mathrm{~h}$ at room temperature, and then incubated overnight at $4^{\circ} \mathrm{C}$ with the following primary antibodies (Abs): Anti-cleaved caspase-3 rabbit monoclonal (m)Ab (1:1,000; cat. no. \#9664; Cell Signaling Technology, Inc., Danvers, MA, USA); anti-cleaved caspase-9 rabbit polyclonal (p)Ab (1:500; cat. no. C7729; Sigma-Aldrich; Merck KGaA); anti-B-cell lymphoma 2 (Bcl-2) rabbit pAb (1:1,000; cat. no. ab196495; Abcam, Cambridge, $\mathrm{UK}$ ); anti-Bcl-2-associated $\mathrm{X}$ protein (Bax) rabbit $\mathrm{mAb}$ (1:2,000; cat. no. ab182733; Abcam); anti-nuclear factor-like 2 (Nrf2) rabbit pAb (1:1,000; cat. no. ab92946; Abcam); anti-APJ rabbit pAb (1:500; cat. no. ab214369; Abcam); anti-apelin rabbit pAb (1:1,000; cat. no. ab125213; Abcam); anti-nicotinamide adenine dinucleotide phosphate (NADPH) oxidase 4 rabbit mAb (1:3,000; cat. no. ab133303; Abcam); anti-Lamin B1 rabbit mAb (1:3,000; cat. no. ab133741; Abcam) and anti- $\beta$-actin rabbit pAb (1:3,000; cat. no. ab8227; Abcam). Following washing with PBS-0.1\% Tween-20 (PBS-T), membranes were incubated with horseradish peroxidase-conjugated goat anti-rabbit secondary antibody (1:8,000; cat. no. ab6721; Abcam) for $1 \mathrm{~h}$ at room temperature. Membranes were then washed three times with PBS-T and visualized using an enhanced chemiluminescence system (Bio-Rad Laboratories, Inc.).

Statistical analysis. All data were presented as the mean \pm standard deviation $(n=3)$. SPSS version 13.0 software (SPSS, Inc., Chicago, IL, USA) was used for data analysis. The normality of data was determined using a 
A

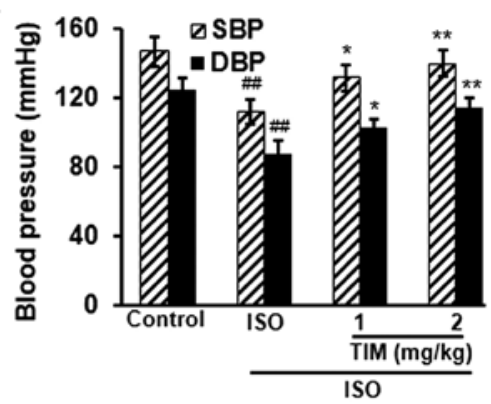

C

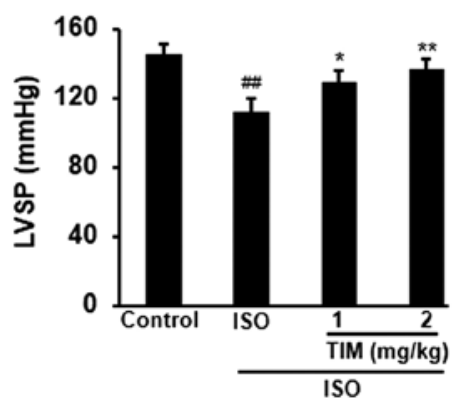

B

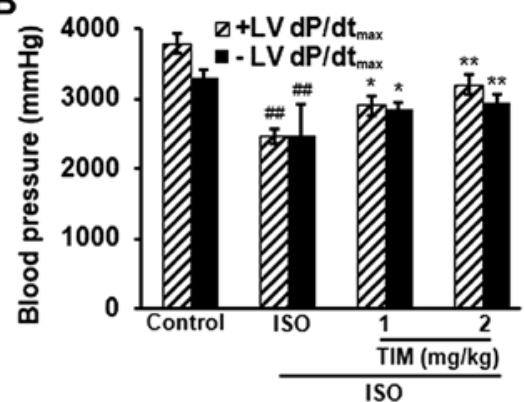

D

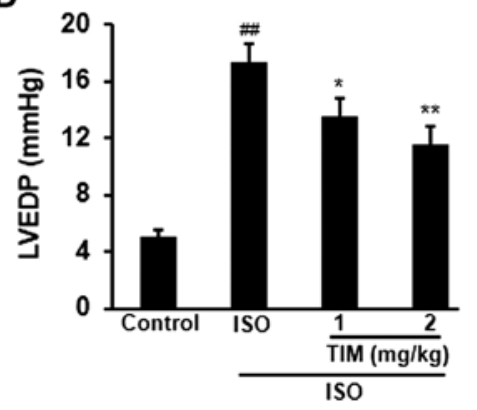

Figure 2. TIM enhances cardiac function following ISO-induced MI in rats. Effects of ISO-induced MI and treatment with TIM on (A) blood pressure, (B) $\pm \mathrm{LV} \mathrm{dP/dt}$ max , (C) LVSP and (D) LVEDP. Data are presented as the mean \pm standard deviation. Samples were measured in triplicate. Data distributions were analyzed using a Kolmogorov-Smirnov test. ${ }^{\# \#} \mathrm{P}<0.01$ vs. control; ${ }^{*} \mathrm{P}<0.05$ and ${ }^{* *} \mathrm{P}<0.01 \mathrm{vs}$. ISO. DBP, diastolic blood pressure; ISO, isoproterenol; $\pm \mathrm{LV}$ $\mathrm{dP} / \mathrm{dt}_{\max }$, maximum left ventricular contraction/relaxation velocity; LVEDP, left ventricular end-diastolic pressure; LVSP, left ventricular systolic pressure; MI, myocardial infarction; SBP, systolic blood pressure; TIM, (3R)-5,6,7-trihydroxy-3-isopropyl-3-methylisochroman-1-one.

Kolmogorov-Smirnov test. Differences between two groups were analyzed using t-tests; differences between $>2$ groups were analyzed using one-way analyses of variance followed by a Dunnett's test. $\mathrm{P}<0.05$ was considered to indicate a statistically significant difference.

\section{Results}

Effects of TIM on the body and heart weights of rats. Rats with ISO-induced MI were treated with low and high doses of TIM. Compared with the control, there were no significant differences in the body weight, heart weight or heart index of rats following ISO or TIM treatment (Fig. 1).

TIM enhances cardiac function following MI in rats. ISO treatment significantly altered cardiac function; the blood pressure, $\pm \mathrm{LV} \mathrm{dP/dt}{ }_{\max }$ and LVSP of ISO rats were significantly decreased compared with the control, whereas the LVEDP was significantly increased (Fig. 2). Conversely, treatment with 1 and $2 \mathrm{mg} / \mathrm{kg}$ TIM significantly increased blood pressure, $\pm \mathrm{LV}$ $\mathrm{dp} / \mathrm{dt}_{\max }$ and LVSP, and decreased the LVEDP of rats compared with ISO treatment alone (Fig. 2).

TIM treatment protects cardiomyocytes against $I S O$-induced $M I$. ISO treatment induced severe damage to cardiomyocytes, as determined by the significant increases in LDH levels, cytochrome-c release and DNA damage compared with the control; however, TIM treatment significantly ameliorated these effects (Fig. 3A-C). Furthermore, ISO treatment significantly increased the activity of caspases, upregulated the expression of cleaved caspase-3, cleaved caspase- 9 and Bax, and downregulated the expression of $\mathrm{Bcl}-2$; treatment with TIM induced the opposite effect (Fig. 3D and E).

Treatment with TIM reduces oxidative stress. Oxidative stress in myocardial tissue was determined by the levels of MDA and GSH, the activity of SOD, and the protein expression of NADPH oxidase 4 and Nrf2. Compared with normal control rats, oxidative stress was significantly induced following ISO treatment, with increased levels of MDA, reduced activity of SOD and decreased levels of GSH (Fig. 4A-C). Conversely, treatment with TIM significantly reduced MDA levels, and increased the levels of GSH and the activity of SOD, when compared with ISO treatment alone. Furthermore, TIM treatment markedly downregulated NADPH oxidase 4, and increased total and nuclear Nrf2 protein expression (Fig. 4D and E).

TIM increased apelin and APJ. Compared with the control, the plasma and myocardial levels of apelin were significantly decreased following ISO treatment; however, treatment with TIM increased apelin levels when compared with ISO treatment alone (Fig. 5A and B). Furthermore, treatment with TIM eliminated the ISO-induced decreases in the expression of apelin and APJ mRNA and protein (Fig. 5C and D).

\section{Discussion}

Numerous medicinal products have been derived from herbal plants (23). TIM is a novel compound isolated from Alpinia katsumadai Hayata that exhibited cardioprotective effects against lipoteichoic acid-induced damage in rat cardiomyoblast cells via the inhibition of oxidative stress (18). 
A
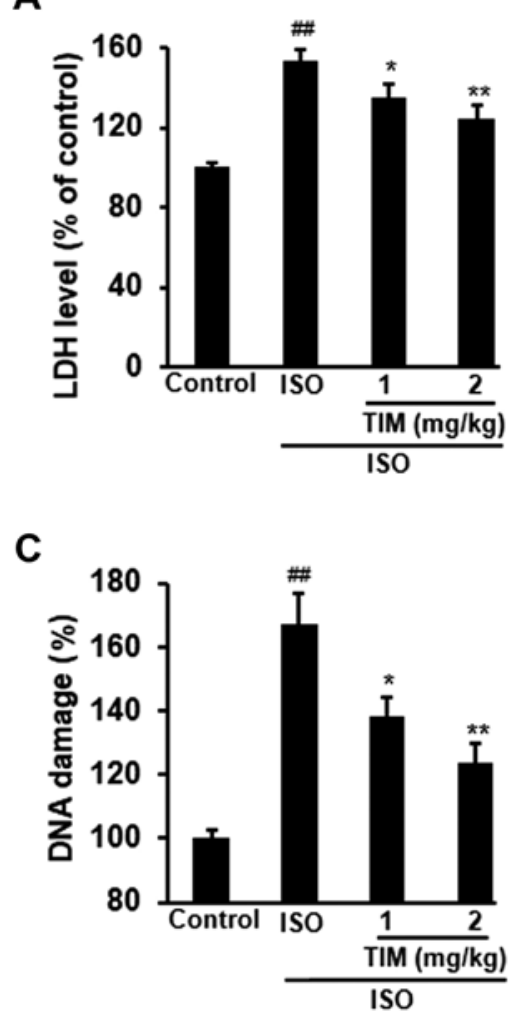

B

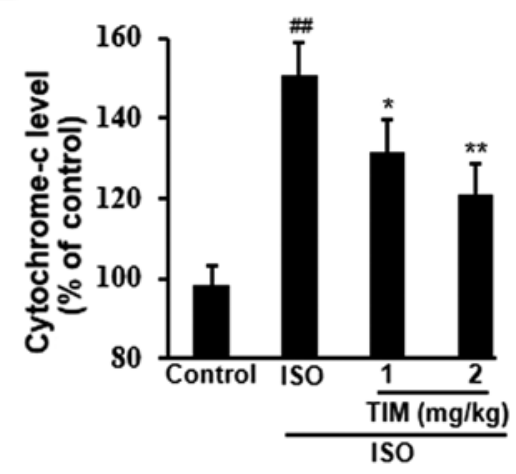

D

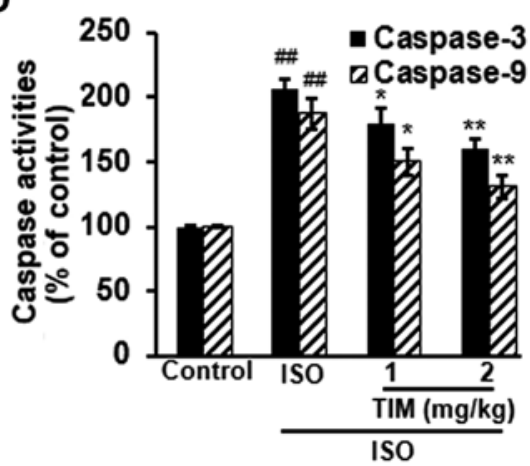

E

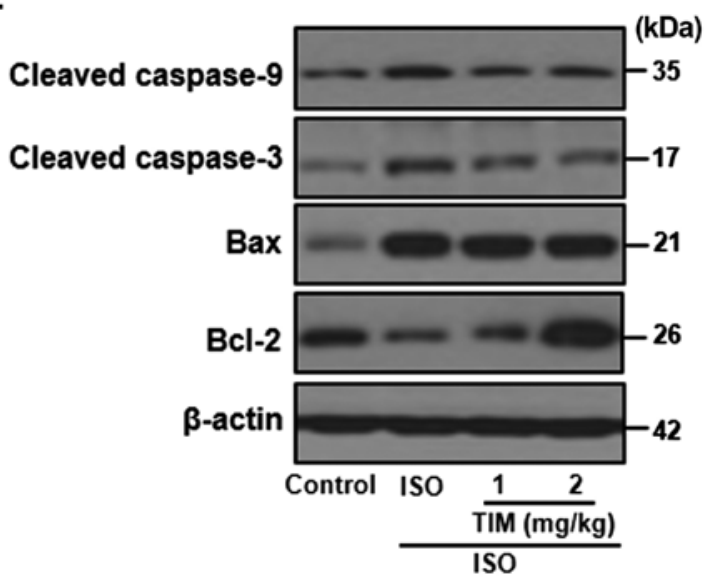

Figure 3. TIM treatment protects cardiomyocytes against ISO-induced MI. Levels of (A) LDH, (B) cytochrome-c, (C) DNA damage and (D) caspase-3/9 activity following ISO-induced MI and treatment with TIM. (E) Representative western blot of cleaved caspases-3 and -9, Bax and Bcl-2 following ISO-induced MI and treatment with TIM. Data are presented as the mean \pm standard deviation. Samples were measured in triplicate. Data distributions were analyzed using a Kolmogorov-Smirnov test. ${ }^{\# \#} \mathrm{P}<0.01$ vs. control; ${ }^{*} \mathrm{P}<0.05$ and ${ }^{* *} \mathrm{P}<0.01$ vs. ISO. Bcl-2, B-cell lymphoma 2; Bax, Bcl-2-associated $\mathrm{X}$ protein; ISO, isoproterenol; LDH, lactate dehydrogenase; MI, myocardial infarction; TIM, (3R)-5,6,7-trihydroxy-3-isopropyl-3-methylisochroman-1-one.

In the present study, it was demonstrated that TIM protected cardiomyocytes against ISO-induced MI, potentially via the apelin/APJ signaling pathway. Injection with ISO induced severe cardiac dysfunction in rats; however, treatment with TIM ameliorated left ventricular contractile dysfunction, as determined by increased blood pressure, $\pm \mathrm{LV} \mathrm{dp} / \mathrm{dt}_{\max }$ and LVSP, and decreased LVEDP. These findings suggested that TIM improved cardiac function in ISO-treated rats.

Cardiomyocyte apoptosis serves an important role in the progression of cardiac dysfunction in acute and long-term settings following MI; apoptosis reduces the number of normal contractile cardiomyocytes, leading to adverse ventricular remodeling $(24,25)$. Drugs that prevent cardiomyocyte apoptosis, including angiotensin II receptor antagonists and $\beta$-blockers, have been reported to be effective in the treatment of heart failure, providing a potential target in the prevention of pathological progression $(26,27)$. As a principal cytotoxic lesion, DNA double-strand breaks are frequently investigated to determine cytotoxicity $(28,29)$. The levels of caspase- 3 and caspase- 9 activity have been used to evaluate apoptosis (30). Additionally, two important members of the Bcl-2 family, Bcl-2 and Bax, are directly associated with the regulation of apoptosis; Bcl-2 inhibits cell apoptosis, whereas Bax promotes apoptosis, and the 

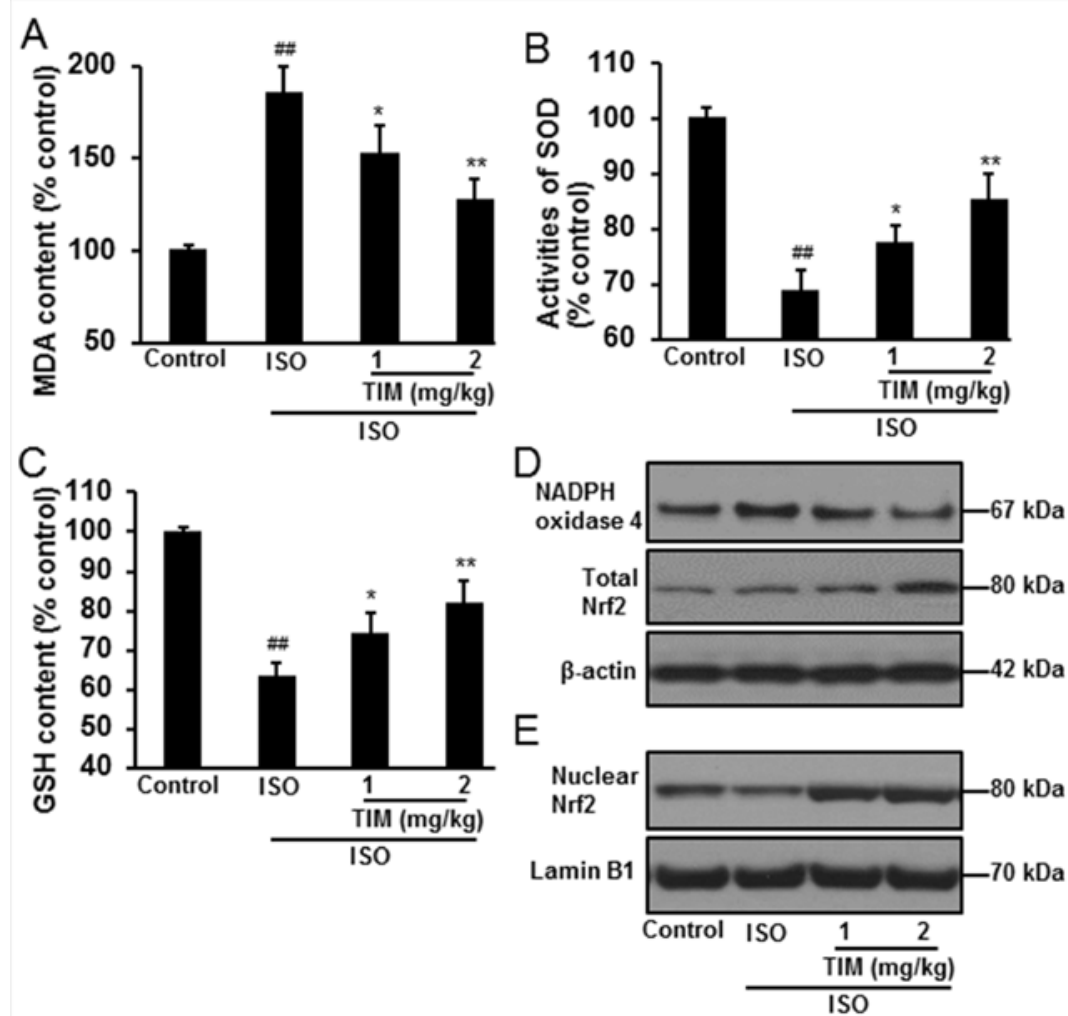

Figure 4. TIM treatment reduces oxidative stress in rat cardiomyocytes following ISO-induced MI. Levels of (A) MDA, (B) SOD activity and (C) GSH following ISO-induced MI and treatment with TIM. (D and E) Representative western blot of NADPH oxidase 4, and total and nuclear Nrf2 protein expression following ISO-induced MI and treatment with TIM. Data are presented as the mean \pm standard deviation. Samples were measured in triplicate. Data distributions were analyzed using a Kolmogorov-Smirnov test. ${ }^{\# \#} \mathrm{P}<0.01$ vs. control; ${ }^{*} \mathrm{P}<0.05$ and ${ }^{* *} \mathrm{P}<0.01$ vs. ISO. GSH, glutathione; ISO, isoproterenol; MDA, malondialdehyde; MI, myocardial infarction; NADPH, nicotinamide adenine dinucleotide phosphate; Nrf2, nuclear factor-like 2; SOD, superoxide dismutase; TIM, (3R)-5,6,7-trihydroxy-3-isopropyl-3-methylisochroman-1-one.

A

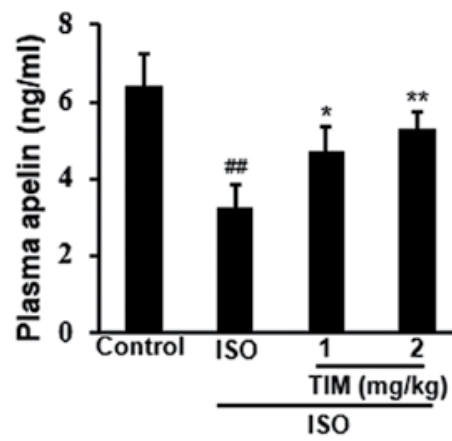

C

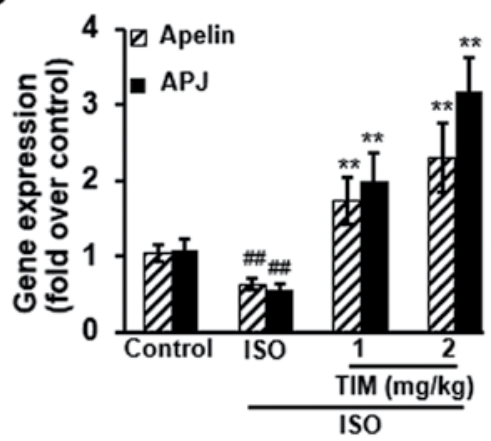

B

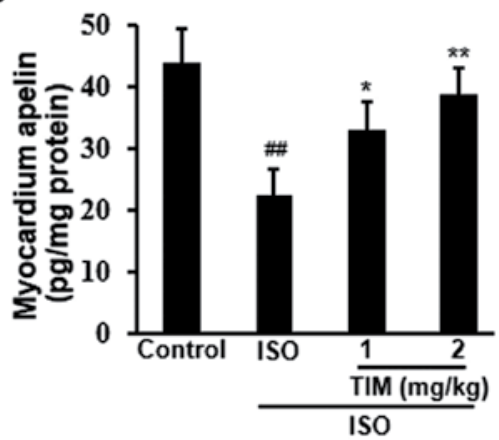

D

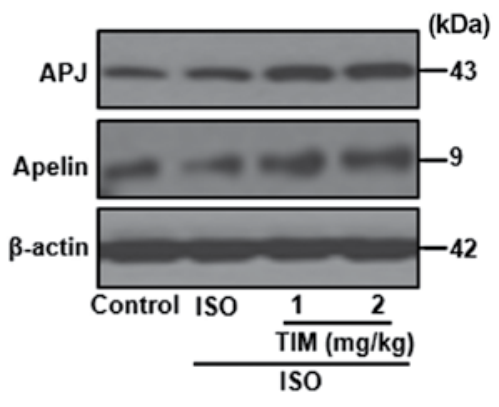

Figure 5. TIM treatment increases the expression of apelin and APJ.(A) Plasma and (B) myocardial levels of apelin following ISO-induced MI and treatment with TIM. Expression of apelin and APJ (C) mRNA and (D) protein following ISO-induced MI and treatment with TIM. Data are presented as the mean \pm standard deviation. Samples were measured in triplicate. Data distributions were analyzed using a Kolmogorov-Smirnov test. ${ }^{\# \#} \mathrm{P}<0.01$ vs. control; ${ }^{*} \mathrm{P}<0.05$ and ${ }^{* * *} \mathrm{P}<0.01$ vs. ISO. APJ, apelin receptor; ISO, isoproterenol; MI, myocardial infarction; TIM, (3R)-5,6,7-trihydroxy-3-isopropyl-3-methylisochroman-1-one. 
Bcl-2/Bax ratio of cells determines their fate following apoptotic stimulation (31-33). In the present study, injecting rats with ISO induced cardiomyocyte damage, characterized by DNA damage, increased levels of caspase-3/9 activity, marked downregulation of $\mathrm{Bcl}-2$ expression and upregulation of Bax expression. By contrast, these ISO-induced effects were ameliorated by TIM treatment, indicating that TIM may protect myocardial cells against apoptosis in vivo.

Increased oxidative stress was observed in the myocardium following treatment with ISO. Oxidative stress affects various biological macromolecules and suppresses cellular functions $(34,35)$. NADPH oxidase 4 is expressed primarily in the mitochondria of cardiac myocytes (36). It was reported that cardiac hypertrophy and apoptosis were attenuated, and improved cardiac function was observed in NADPH oxidase 4-deficient mice compared with wild-type mice in a pressure overload model (37). Conversely, overexpression of NADPH oxidase 4 in mouse heart tissue exacerbated cardiac dysfunction, fibrosis and apoptosis in response to pressure overload, indicating that NADPH oxidase 4 was a major source of oxidative stress in the failing heart, thereby mediating mitochondrial and cardiac dysfunction (37). Oxidative stress-induced damage has been hypothesized to be a major pathogenic mechanism underlying numerous disorders, and previous studies have reported that supplementation of external antioxidants may be an effective strategy to maintain the balance between antioxidative and intracellular oxidative systems $(38,39)$. Nrf2 is important in cell defense against oxidative stress; it is inactive in the cytoplasm when bound to Kelch-like ECH-associated protein 1 (Keap1), but is released from Keap1 upon activation and moves into the cell nucleus (40). Nrf2 then binds with antioxidant response elements and induces the expression of cytoprotective targets, including antioxidant proteins, phase II detoxifying enzymes and molecular proteasome/chaperones (41). In the present study, treatment with TIM upregulated cytoplasmic and nuclear Nrf2 expression in cardiac tissue, which was accompanied by reductions in MDA levels and the protein expression of NADPH oxidase 4 , and increased SOD activity and GSH levels. These results indicated that TIM may induce antioxidative gene expression to restore oxidative homeostasis.

Identified as an endogenous ligand of the G-protein-coupled receptor APJ, apelin is expressed in various tissues, including the heart, where it exhibits potent hypotensive and positive inotropic properties, inducing endothelium- and nitric oxide-dependent vasodilatation $(42,43)$. It was previously revealed that apelin-deficient mice developed progressive heart failure; however, exogenous administration of apelin exerted inotropic effects on animals (44). These findings were consistent with previous clinical observations that revealed that a disturbance in the endogenous apelin/APJ signaling pathway is associated with cardiac dysfunction in humans (45), indicating that the apelin/APJ pathway serves an important role in regulating cardiovascular homeostasis. An increasing body of evidence has indicated that apelin/APJ signaling functions as a critical mediator of cardiovascular homeostasis and is involved in the pathophysiology of cardiovascular diseases; targeting the apelin/APJ axis promotes cardioprotection against cardiovascular diseases $(46,47)$. The results of the present study demonstrated that apelin levels were significantly decreased in the plasma and myocardium following ISO treatment; however, TIM treatment produced the opposite effects, and increased the mRNA and protein expression of apelin and APJ. These findings indicated that TIM may improve cardiac function via activation of the apelin/APJ signing pathway.

In conclusion, it was demonstrated that TIM exerted cardioprotective effects in a rat model of ISO-induced MI, ameliorating cardiac dysfunction and inhibiting cardiomyocyte apoptosis. These effects may have been mediated at least partially via the apelin/APJ signaling pathway. These findings provide evidence for the development of TIM as a therapeutic agent in the treatment of MI.

\section{Acknowledgements}

The authors thank Professor Lin from Shantou University Medical College (Shantou, China) for providing TIM in this study.

\section{Funding}

The present study was financially supported by the Department of Science and Technology of Jilin Province (grant no. 20170622012JC).

\section{Availability of data and materials}

The datasets used and/or analyzed during the current study are available from the corresponding author on reasonable request.

\section{Authors' contributions}

PY and HY made substantial contributions to the conception and design of the study. Experiments were performed and analyzed by MD, LG and CZ. The manuscript was drafted by MD, PY and HY.

\section{Ethics approval and consent to participate}

All animal experiments were performed in accordance with the Chinese Legislation on the Use and Care of Laboratory Animals, and approved by the Ethical Committee on Animal Care and Use of Jilin University.

\section{Patient consent for publication}

Not applicable.

\section{Competing interests}

The authors declare that they have no competing interests.

\section{References}

1. Bogomolov AN, Kozlov KL, Kurochkina ON and Olesiuk IB: Coronary stenting in elderly patients with acute myocardial infarction (review). Adv Gerontol 26: 151-160, 2013 (In Russian).

2. Wartenberg KE: Malignant middle cerebral artery infarction. Curr Opin Crit Care 18: 152-163, 2012.

3. Yang G, Min D, Yan J, Yang M and Lin G: Protective role and mechanism of snakegourd peel against myocardial infarction in rats. Phytomedicine 42: 18-24, 2018 
4. Bakhta O, Blanchard S, Guihot AL, Tamareille $S$ Mirebeau-Prunier D, Jeannin P and Prunier F: Cardioprotective role of colchicine against inflammatory injury in a rat model of acute myocardial infarction. J Cardiovasc Pharmacol Ther 23: 446-455, 2018

5. Senthil S, Chandramohan G and Pugalendi KV: Isomers (oleanolic and ursolic acids) differ in their protective effect against isoproterenol-induced myocardial ischemia in rats. Int J Cardiol 119: 131-133, 2007.

6. Grimm D, Elsner D, Schunkert H, Pfeifer M, Griese D, Bruckschlegel G, Muders F, Riegger GA and Kromer EP: Development of heart failure following isoproterenol administration in the rat: Role of the renin-angiotensin system. Cardiovasc Res 37: 91-100, 1998

7. Zhang GX, Kimura S, Nishiyama A, Shokoji T, Rahman M, Yao L, Nagai Y, Fujisawa Y, Miyatake A and Abe Y: Cardiac oxidative stress in acute and chronic isoproterenol-infused rats. Cardiovasc Res 65: 230-238, 2005.

8. Wang SB, Tian S, Yang F, Yang HG, Yang XY and Du GH: Cardioprotective effect of salvianolic acid A on isoproterenol-induced myocardial infarction in rats. Eur J Pharmacol 615: 125-132, 2009.

9. Zhou R, He LF, Li YJ, Shen Y, Chao RB and Du JR: Cardioprotective effect of water and ethanol extract of Salvia miltiorrhiza in an experimental model of myocardial infarction. J Ethnopharmacol 139: 440-446, 2012.

10. Padmanabhan $M$ and Prince PSM: Effects of pharmacological amounts of S-allylcysteine on lipids in normal and isoproterenol-induced myocardial infarction in rats. J Sci Food Agric 86: 772-777, 2006.

11. Cheng J, Luo X, Huang Z and Chen L: Apelin/APJ system: A potential therapeutic target for endothelial dysfunction-related diseases. J Cell Physiol: Dec 26, 2018 (Epub ahead of print).

12. Tatemoto K, Hosoya M, Habata Y, Fujii R, Kakegawa T, Zou MX, Kawamata Y, Fukusumi S, Hinuma S, Kitada C, et al: Isolation and characterization of a novel endogenous peptide ligand for the human APJ receptor. Biochem Biophys Res Commun 251: 471-476, 1998

13. Chong KS, Gardner RS, Morton JJ, Ashley EA and McDonagh TA: Plasma concentrations of the novel peptide apelin are decreased in patients with chronic heart failure. Eur J Heart Fail 8: 355-360, 2006.

14. Chen MM, Ashley EA, Deng DX, Tsalenko A, Deng A, Tabibiazar R, Ben-Dor A, Fenster B, Yang E, King JY, et al: Novel role for the potent endogenous inotrope apelin in human cardiac dysfunction. Circulation 108: 1432-1439, 2003.

15. Tao J, Zhu W, Li Y, Xin P, Li P, Liu M, Li J, Redington AN and Wei M: Apelin-13 protects the heart against ischemia-reperfusion injury through inhibition of ER-dependent apoptotic pathways in a time-dependent fashion. Am J Physiol Heart Circ Physiol 301: H1471-H1486, 2011.

16. Kang K, Tarchick MJ, Yu X, Beight C, Bu P and Yu M: Carnosic acid slows photoreceptor degeneration in the Pde6b(rd10) mouse model of retinitis pigmentosa. Sci Rep 6: 22632, 2016.

17. Wu YZ, Qiao F, Xu GW, Zhao J, Teng JF, Li C and Deng WJ: Neuroprotective metabolites from the endophytic fungus Penicillium citrinum of the mangrove Bruguiera gymnorrhiza. Phytochem Lett 12: 148-152, 2015.

18. Liu Z, Xie L, Bian T, Qi G and Wang Z: (3R)-5,6,7trihydroxy-3-isopropyl-3-methylisochroman-1-one reduces lipoteichoic acid-induced damage in rat cardiomyoblast cells. Anatol J Cardiol 19: 198-204, 2018.

19. Ministry of Science and Technology. Current Laboratory Animal Laws Regulations, Policies and Administration in China, 2018.

20. Chen DY, Yang F and Lin YQ: Neuroprotective constituent from the seeds of Alpinia katsumadai Hayata. Phytochem Lett 18: 59-63, 2016.

21. Mondal MI and Yeasmin MS: Toxicity study of food-grade carboxymethyl cellulose synthesized from maize husk in Swiss albino mice. Int J Biol Macromol 92: 965-971, 2016.

22. Livak KJ and Schmittgen TD: Analysis of relative gene expression data using real-time quantitative PCR and the 2(-Delta Delta C(T)) method. Methods 25: 402-408, 2001

23. Li L, Zhou X, Li N, Sun M, Lv J and Xu Z: Herbal drugs against cardiovascular disease: Traditional medicine and modern development. Drug Discov Today 20: 1074-1086, 2015.

24. Wang X, Wang Q, Guo W and Zhu YZ: Hydrogen sulfide attenuates cardiac dysfunction in a rat model of heart failure: A mechanism through cardiac mitochondrial protection. Biosci Rep 31: 87-98, 2011.
25. Abbate A, Biondi-Zoccai GG, Bussani R, Dobrina A, Camilot D, Feroce F, Rossiello R, Baldi F, Silvestri F, Biasucci LM and Baldi A: Increased myocardial apoptosis in patients with unfavorable left ventricular remodeling and early symptomatic post-infarction heart failure. J Am Coll Cardiol 41: 753-760, 2003.

26. Ahmet I, Krawczyk M, Heller P, Moon C, Lakatta EG and Talan MI: Beneficial effects of chronic pharmacological manipulation of beta-adrenoreceptor subtype signaling in rodent dilated ischemic cardiomyopathy. Circulation 110 : 1083-1090, 2004.

27. Soga M, Kamal FA, Watanabe K, Ma M, Palaniyandi S, Prakash P, Veeraveedu P, Mito S, Kunisaki M, Tachikawa H, et al: Effects of angiotensin II receptor blocker (candesartan) in daunorubicin-induced cardiomyopathic rats. Int J Cardiol 110: 378-385, 2006.

28. Yuan J, Adamski R and Chen J: Focus on histone variant H2AX: To be or not to be. FEBS Lett 584: 3717-3724, 2010.

29. Kuo LJ and Yang LX: Gamma-H2AX-a novel biomarker for DNA double-strand breaks. In Vivo 22: 305-309, 2008.

30. Xin BR, Liu JF, Kang J and Chan WP: (2R, 3S)-pinobanksin3-cinnamate, a new flavonone from seeds of Alpinia galanga willd., presents in vitro neuroprotective effects. Mol Cell Toxicol 10: 165-172, 2014

31. Kirkland RA and Franklin JL: Bax, reactive oxygen, and cytochrome c release in neuronal apoptosis. Antioxid Redox Signal 5: 589-596, 2003.

32. Brunelle JK and Letai A: Control of mitochondrial apoptosis by the Bcl-2 family. J Cell Sci 122: 437-441, 2009.

33. Cory S and Adams JM: The Bcl2 family: Regulators of the cellular life-or-death switch. Nat Rev Cancer 2: 647-656, 2002.

34. Adibhatla RM and Hatcher JF: Lipid oxidation and peroxidation in CNS health and disease: From molecular mechanisms to therapeutic opportunities. Antioxid Redox Signal 12: 125-169, 2010.

35. Wang $X$ and Michaelis EK: Selective neuronal vulnerability to oxidative stress in the brain. Front Aging Neurosci 2: 12, 2010.

36. Kuroda J and Sadoshima J: NADPH oxidase and cardiac failure. J Cardiovasc Transl Res 3: 314-320, 2010.

37. Kuroda J, Ago T, Matsushima S, Zhai P, Schneider MD and Sadoshima J: NADPH oxidase 4 (Nox4) is a major source of oxidative stress in the failing heart. Proc Natl Acad Sci USA 107: 15565-15570, 2010.

38. Halliwell B: Oxidative stress and neurodegeneration: Where are we now? J Neurochem 97: 1634-1658, 2006.

39. Birben E, Sahiner UM, Sackesen C, Erzurum S and Kalayci O: Oxidative stress and antioxidant defense. World Allergy Organ J 5: 9-19, 2012.

40. Itoh K, Wakabayashi N, Katoh Y, Ishii T, Igarashi K, Engel JD and Yamamoto M: Keap1 represses nuclear activation of antioxidant responsive elements by Nrf2 through binding to the amino-terminal Neh2 domain. Genes Dev 13: 76-86, 1999.

41. Kobayashi $M$ and Yamamoto $M$ : Nrf2-Keap1 regulation of cellular defense mechanisms against electrophiles and reactive oxygen species. Adv Enzyme Regul 46: 113-140, 2006.

42. Medhurst AD, Jennings CA, Robbins MJ, Davis RP, Ellis C, Winborn KY, Lawrie KW, Hervieu G, Riley G, Bolaky JE, et al: Pharmacological and immunohistochemical characterization of the APJ receptor and its endogenous ligand apelin. J Neurochem 84: 1162-1172, 2003.

43. Berry MF, Pirolli TJ, Jayasankar V, Burdick J, Morine KJ, Gardner TJ and Woo YJ: Apelin has in vivo inotropic effects on normal and failing hearts. Circulation 110 (11 Suppl 1): II187-II193, 2004

44. Kuba K, Zhang L, Imai Y, Arab S, Chen M, Maekawa Y, Leschnik M, Leibbrandt A, Markovic M, Schwaighofer J, et al: Impaired heart contractility in Apelin gene-deficient mice associated with aging and pressure overload. Circ Res 101: e32-e42, 2007.

45. Japp AG and Newby DE: The apelin-APJ system in heart failure: Pathophysiologic relevance and therapeutic potential. Biochem Pharmacol 75: 1882-1892, 2008.

46. He L, Xu J, Chen L and Li L: Apelin/APJ signaling in hypoxia-related diseases. Clin Chim Acta 451: 191-198, 2015.

47. Yu XH, Tang ZB, Liu LJ, Qian H, Tang SL, Zhang DW, Tian GP and Tang CK: Apelin and its receptor APJ in cardiovascular diseases. Clin Chim Acta 428: 1-8, 2014. 